\title{
Caveolin-I and -2 regulate cell motility in castration-resistant prostate cancer
}

This article was published in the following Dove Press journal: Research and Reports in Urology

\section{Toyoharu Kamibeppu \\ Koji Yamasaki \\ Kozue Nakahara \\ Takahiro Nagai \\ Naoki Terada \\ Hiromasa Tsukino \\ Shoichiro Mukai \\ Toshiyuki Kamoto}

Department of Urology, Faculty of Medicine, University of Miyazaki, Kiyotake, Japan
Correspondence: Shoichiro Mukai Department of Urology, Faculty of Medicine, University of Miyazaki, 5200 Kihara, Kiyotake, Miyazaki 889-1692, Japan

Tel +81985852968

Fax +8I 985856958

Email syoichiro_mukai@med.miyazaki-u. ac.jp
Background: Caveolin (Cav)-1 and Cav-2 are cell membrane proteins, which are structural proteins of caveolae and are reported to be positive regulators of cell survival and metastasis in prostate cancer (PC). In a previous study, we reported that elevated levels of Cav-1 and Cav-2 were significantly associated with PC progression. However, their functions in $\mathrm{PC}$ have not yet been clarified. In this study, we examined the function of Cav-1 and Cav-2 in PC cell invasiveness and motility.

Materials and methods: We introduced Cav-1- and Cav-2-specific small interfering into PC3 cells to knock-down (KD) both molecules. We also performed cell proliferation assay, wound healing assay, migration assay, and invasion assay using PC3 cells and compared the results between Cav-1-KD, Cav-2-KD, and negative control PC3 cells. In addition, we performed real-time quantitative PCR (RT-qPCR) and $\mathrm{RT}^{2}$ Profiler PCR Array analysis to identify factors influencing migration.

Results: We observed no significant difference in the proliferative and invasive activities of Cav-1-KD and Cav-2-KD PC3 cells; however, the cell motility was significantly decreased compared with negative control PC3 cells. RT-qPCR revealed that the expression of vimentin and N-cadherin was downregulated in Cav-1-KD PC3 cells. In addition, PCR array revealed a decreased expression of MGAT5, MMP13, and MYCL in Cav-1-KD PC3 and ETV4, FGFR4, and SRC in Cav-2-KD PC3.

Conclusion: Cav-1 and Cav-2 may positively contribute to the upregulation of castrationresistant PC cell migration. Cav-induced regulation of several molecules including vimentin, N-cadherin, MGAT5, MMP13, MYCL, ETV4, FGFR4, and SRC may have an important role in PC3 cell motility. However, further examination will be required.

Keywords: caveolin-1, caveolin-2, CRPC, cell motility

\section{Introduction}

Prostate cancer (PC) is the most commonly diagnosed cancer in men and is the second leading cause of death due to cancer in men in developed countries. ${ }^{1}$ Localized PCs are usually treated by radical prostatectomy or radiation. In Japan, $21 \%$ of PC patients present with distant metastases and $19 \%$ of PC patients present with locally advanced disease at diagnosis. ${ }^{2}$ Androgen deprivation therapy (ADT) is a mainstay of treatment for locally advanced and metastatic PC. ADT is reported to be effective for 3-5 years, ${ }^{3,4}$ although medically or surgically castrated PC eventually transforms into castration-resistant PC (CRPC), and the prognosis of the patients in the CRPC stage is poor. Advanced CRPC is a multifaceted problem and needs many approach. CRPC is 
associated with several factors, including downregulation of performance status and presence of bone pain. Bone metastases will develop in $90 \%$ of men with CRPC and can produce significant morbidity, including pain, pathologic fractures, spinal cord compression, and bone marrow failure. Biological effects are also common, including anemia, weight loss, fatigue, hypercoagulability, and infection. ${ }^{5}$ Supportive care and maintenance of the quality of life remain the priority. We must build on what is currently available to further improve the outcome in these poor prognosis patients.

Caveolins (Cavs) are well-known principal components of caveolae, which are involved in cellular process including endocytosis, lipid homeostasis, and signal transduction as physiological functions. ${ }^{6,7}$ Caveolae are mainly expressed in fibroblasts, vascular endothelial cells, adipocytes, and epithelial cells. ${ }^{6-8}$ The Cav family is composed of three isoforms, namely, Cav-1, Cav-2, and Cav-3. ${ }^{6-8}$ Although Cav-1 and Cav-2 are expressed in various tissues, Cav-3 has been reported to be expressed in skeletal tissue, cardiac myocytes, and smooth muscle cells. ${ }^{6-9}$ All three Cavs show basic structural motif of N-terminal Cav-1 scaffolding domain (CSD), transmembrane domain, and C-terminal domain. ${ }^{6-8} \mathrm{Cav}-1$ is a $22 \mathrm{kDa}$ protein composed of 178 amino acids and has been most sufficiently investigated in Cav family. ${ }^{6,7,9} \mathrm{Cav}-1$ reported to be expressed predominantly in terminally differentiated cells including adipocytes, endothelial cells, and smooth muscle cells. ${ }^{6-9}$ The role of Cav-1 in cholesterol trafficking and homeostasis is reported to be one of the major physiological functions. ${ }^{6-9}$

The function of Cavs in cancer is controversial because the activity is reported to differ by cancer cell types. ${ }^{10,11}$ Downregulation of Cav-1 was reported in the carcinomas of breast, ${ }^{12}$ colon, ${ }^{13}$ ovary, ${ }^{14}$ and sarcoma; ${ }^{15}$ the locus encoding Caveolin-1 and Caveolin-2 genes is reported to be deleted in several cancer cells. ${ }^{16}$ In contrast, overexpression of Cav-1 was observed in prostate, ${ }^{17-20}$ pancreas, ${ }^{21,22}$ colon, ${ }^{23}$ breast, ${ }^{24}$ and esophagus carcinoma ${ }^{25}$ and increased expression of Cav-2 was observed in esophageal carcinoma, ${ }^{26,27}$ urothelial carcinoma, ${ }^{28}$ and several cancer cell lines including glioma, cervical cancer, lung adenocarcinoma, and breast cancer. ${ }^{7}$ In addition, promotion of chemoresistance in gastric cancer and lymph nodes metastasis in non-small-cell lung cancer by Cav-1 was also reported. ${ }^{29,30}$ Interestingly, downregulation of Cav-1 led to the conversion of androgen-insensitive metastatic mouse PC cells (malignant phenotype) to androgen-sensitive phenotype. ${ }^{31}$

In the previous work, we reported that plasma concentration of Cav-1 and Cav-2 was increased in CRPC patients compared with androgen-sensitive PC patients. ${ }^{17}$ According to the results, we analyzed the function of Cav-1 and Cav-2 in CRPC cell line PC3.

\section{Materials and methods Cell culture}

Three androgen-independent PC cell lines (PC3, DU145, and 22Rv1), a androgen-dependent PC cell line (LNCaP), and Hela cell line were obtained from American Type Culture Collection (ATCC) (Manassas, VA, USA), cultured in $10 \mathrm{~cm}$ in diameter cell culture dishes with DMEM (Thermo Fisher Scientific, Waltham, MA, USA) containing 10\% (v/v) FBS (Thermo Fisher Scientific) at $37^{\circ} \mathrm{C}$ in a humidified atmosphere of $5 \% \mathrm{CO}_{2}$.

\section{RNA extraction and real-time qRT-PCR analysis}

Total cellular RNA was extracted from cells using an RNA Mini kit (Ambion, Paisley, OR, USA). For qRT-PCR, $3 \mu \mathrm{g}$ of total RNA was reverse transcribed with a mixture of oligo(dT) and random primers and processed for each PCR reaction as described previously. ${ }^{17}$ The primers used for real-time qRTPCR were as follows:

Cav-1 forward, 5'-TTCTGGGCTTCATCTG GCAAC3'; reverse, 5'-GCTCAGCCCTATTGGTCCACTTTA-3' (93 bp);

Cav-2 forward, 5'-CACCCTCAGCTGTCTGCACAT-3'; reverse, 5'-GGCAGAACCATTAGGCAGGTCTT-3' (66 bp);

Glyceraldehyde-3-phosphate dehydrogenase (GAPDH) forward, 5'-GCACCGTCAAGGCTGAGAAC-3'; reverse, 5'-TGGTGAAGACGCCAGTGGA-3' (138 bp);

Androgen receptor (AR) forward, 5'-TCCATTGCCCACCAAAGACTA-3'; reverse, 5'-GCAAATCTGGCCTGTCACCTC-3' (150 bp);

E-cadherin forward, 5'-AAGTGCTGCAGCCAAAGACAGA-3'; reverse, 5'-AAATTGCCAGGCTCAATGACAAG-3' (84 bp);

$\mathrm{N}$-cadherin forward, 5'-CGAATGGATGAAA GACCCATCC-3'; reverse, 5'-GCCACTGCCTTCATAGTCAAACACT-3' (171 bp);

Vimentin forward, 5'-AACCTGGCCGAGGACATCA-3'; reverse, 5'-TCAAGGTCAAGACGTGCCAGA-3' (134 bp);

Hepatocyte growth factor activator inhibitor type 1 (HAI1) forward, 5'-GGTGACACGGATGTCAGGGTA-3'; reverse 5'-CACTGTCAGCTGGAACAGGTAGG-3' (93 bp); 
Hepatocyte growth factor activator inhibitor type 2 (HAI2) forward, 5'-GACGGAAACAGCAATAATTACCTGA-3'; reverse, 5'-TTGAACATATCGCTGGAGTGGTC-3' (170 bp);

EGFR forward, 5'-TTGCCAAGGCACGAGTAACAAG-3'; reverse, 5'-CCACTGTGTTGAGGGCAATGA$3^{\prime}$ (200 bp);

SNAIL forward, 5'-GACCACTATGCCGCGCTCTT-3'; reverse, 5'-TCGCTGTAGTTAGGCTTCCGATT-3' (69 bp);

SNAIL2 forward, 5'-TTTCCAGACCCTGGTTGCTTC-3'; reverse, 5'-CTCAGATTTGACCTGTCTGC AAATG-3'.

Real-time qRT-PCRs were performed using a Thermal Cycler Dice Real-Time System II (Takara Bio, Shiga, Japan). Reaction mixtures $(25 \mu \mathrm{L})$ containing $2 \mu \mathrm{L}$ of cDNA template, $1 \mu \mathrm{L}$ of each sense and antisense primers, and $1 \times$ SYBR Premix Ex Taq II (Takara Bio) were amplified as follows: $95^{\circ} \mathrm{C}$ for 30 seconds and 40 cycles at $95^{\circ} \mathrm{C}$ for 5 seconds, $60^{\circ} \mathrm{C}$ for 30 seconds, and a final dissociation stage $\left(95^{\circ} \mathrm{C}\right.$ for 15 seconds, $60^{\circ} \mathrm{C}$ for 30 seconds, and $95^{\circ} \mathrm{C}$ for 15 seconds). GAPDH was used as an internal control. The results were evaluated using the Thermal Cycler Dice Real-Time System software program. The $\Delta \Delta^{-\mathrm{Ct}}$ algorithm was used to analyze the relative changes in gene expression.

\section{Protein extraction immunoblot analysis}

The cells were washed twice with ice-cold PBS followed by the addition of $1.5 \mathrm{~mL}$ of $10 \%$ trichloroacetic acid on ice. The degenerated cells were scraped and collected into microcentrifuge tubes and centrifuged at $14,000 \mathrm{rpm}$ at $4^{\circ} \mathrm{C}$ for 3 minutes. The pellet was dissolved in an extraction solution consisting of $7 \mathrm{M}$ urea, 2\% Triton-X-100, and 5\% 2-mercaptoethanol. The extracted protein was analyzed by immunoblot analyses. The reaction samples were mixed with SDS-PAGE sample buffer and heated for 15 minutes at $75^{\circ} \mathrm{C}$. SDS-PAGE was performed under reducing conditions using 4-12\% gradient gels. After electrophoresis, the sample proteins were transferred electrophoretically to Immobilon membranes (EMD Millipore, Billerica, MA, USA). After blocking the nonspecific binding site with $5 \%$ nonfat dry milk in $50 \mathrm{mM}$ Tris- $\mathrm{HCl}$ (pH 7.5), $150 \mathrm{mM} \mathrm{NaCl}$, and $0.05 \%$ Tween 20, the membranes were incubated with primary antibody in buffer containing $1 \% \mathrm{BSA}$ at $4^{\circ} \mathrm{C}$ overnight followed by four washes with the buffer and incubation with peroxidase-conjugated secondary antibody diluted in the buffer with $1 \%$ BSA for 1 hour at room temperature. The labeled proteins were visualized with chemiluminescence reagent (PerkinElmer Inc., Waltham, MA, USA).

\section{Knock-down (KD) of Cav-I and Cav-2 in PC3 cells}

Twenty-four hours before siRNA transfection, PC3 cells in the logarithmic phase were inoculated in six-well plates at $5 \times 10^{4}$ cells/well. siRNA ( $20 \mathrm{nM}$ final concentration) was mixed with Lipofectamine RNAiMAX (Thermo Fisher Scientific) in serum-free medium (Opti-MEM; Thermo Fisher Scientific), then incubated for 20 minutes at room temperature, and added to the cells. Transfection was carried out for 48 hours using the Cav-1-specific siRNA (Silencer Select siRNA Lot \# AS00RPF7; Thermo Fisher Scientific), Cav2-specific siRNA (Silencer Select siRNA Lot \# AS00RPF6; Thermo Fisher Scientific), and negative control (NC) siRNA (Silencer Select siRNA Lot \# AS000AAR; Thermo Fisher Scientific). Then, we prepared Cav-1-KD PC3, Cav-2-KD PC3, and si-Negative PC3 cell lines.

\section{Invasion and migration assay}

Twenty-four hours from transfection, Cav-1-KD PC3, Cav2-KD PC3, and si-Negative PC3 cell lines were used for both cell migration and cell invasion assay. Cell migration assay was performed utilizing Culturex 96 well Cell Migration Assay Kit including boyden chamber consisting of polyethylene terephthalate membrane with $8 \mu \mathrm{m}$ pores (Trevigen Inc., Gaithersburg, MD, USA), according to manufacturer's instructions. Briefly, each cell was serum starved for 20 hours and 25,000 cells were seeded to top chamber. After 72 hours culture, cell dissociation solution and Calcein-AM (fluorescent dye) were added and incubated for 1 hour at $37^{\circ} \mathrm{C}$ in $\mathrm{CO}_{2}$ incubator. Then, the top chamber was removed and the plate was read at $485 \mathrm{~nm}$ excitation and $520 \mathrm{~nm}$ emission.

Cell invasion was estimated using a 96-well BME (basement membrane extract) Cell Invasion Assay Kit (Trevigen Inc.) in accordance with the manufacturer's protocol. In brief, each cell was serum starved for 20 hours and 25,000 cells were seeded to top invasion chamber, which was coated by BME solution. The chamber was incubated at $37^{\circ} \mathrm{C}$ in $\mathrm{CO}_{2}$ incubator for 72 hours. Next, cell dissociation solution and Calcein-AM were added. After incubation for 1 hour, the top chamber was removed and the plate was read at $485 \mathrm{~nm}$ excitation and $520 \mathrm{~nm}$ emission.

Using standard curves, measured relative fluorescence units were converted in to cell number to determine the percentage of migration or invasion cells.

\section{Wound healing assay}

Twenty-four hours after transfection, Cav-1-KD PC3, Cav2-KD PC3, and si-Negative PC3 cell lines were used for 
wound healing assay. Cells were harvested at $80 \%$ confluency and resuspended at $5 \times 10^{5}$ cells $/ \mathrm{mL}$. A total of $500 \mu \mathrm{L}$ of cell suspension was added to a 24-well plate, into which a wound field insert was placed (CytoSelect 24-Well Wound Healing Assay; Cell Biolabs, Inc., San Diego, CA, USA). After 24 hours incubation, wound field insert was carefully removed and the wound field area was measured at start and 4 hours. The percent closure was determined as follows: percentage of closure $(\%)=$ migrated cell surface area/total surface area $\times 100$. The closure rate of each cell line was compared using Mann-Whitney $U$ test.

\section{MTS assay}

Twenty-four hours after transfection, Cav-1-KD PC3, Cav2-KD PC3, and si-Negative PC3 cell lines were incubated, after which cell proliferation was measured by MTS assay (Promega Corporation, Fitchburg, WI, USA). Cell proliferation was measured at 0 hours, 24 hours, 48 hours, and 72 hours. Responses to all treatments were assayed in triplicate, and results were expressed as the mean of three separate experiments.

\section{PCR array}

To determine the molecules affected by Cav- 1 and Cav- 2 expressions, which promotes PC3 cell motility, RT² Profiler PCR Array system (Catalog No PAHS-028Z; Qiagen NV, Venlo, the Netherlands) was used. According to the manufacturer's protocol, cDNA from Cav-1-KD PC3, Cav-2-KD $\mathrm{PC} 3$, and si-Negative $\mathrm{PC} 3$ was mixed with $\mathrm{RT}^{2} \mathrm{SYBR}^{\circledR}$ Green qPCR Master Mix (Catalog No 330529; Qiagen NV) and $\mathrm{H}_{2} \mathrm{O}$. Subsequently, $25 \mu \mathrm{L}$ of the mixture was placed into each well of the PCR array (96-well plate was used). The three steps of the cycling program were $96^{\circ} \mathrm{C}$ for 15 seconds, $55^{\circ} \mathrm{C}$ for 40 seconds, and $72^{\circ} \mathrm{C}$ for 30 seconds. The process was repeated for 40 cycles. The threshold cycle changes (delta $\mathrm{Ct}$ ) denote the difference in $\mathrm{Ct}$ for the gene of interest based on the $\mathrm{Ct}$ level of $\beta$-actin within the sample. Relative expression intensity was obtained by calculating 2-delta $\mathrm{Ct}$ for each sample from both stroke and normal participants for further Student's $t$-test analysis. Changes in mRNA expression were compared between each cell line, and the results were reported as fold change: twofold or greater change was considered significant change.

\section{Statistical analysis}

Statistical analysis was performed using SPSS statistics, Version 22 (IBM Corporation, Armonk, NY, USA). Significance was set at $P<0.05$.

\section{Results}

\section{Expression of Cav-I and Cav-2 in PC cell lines}

We investigated the expression of Cavs' mRNA and protein in human PC cell lines by real-time real-time quantitative PCR (RT-qPCR) and immunoblot analysis. High expression of Cav-1 was observed in PC3; however, LNCap and 22Rv1 were not expressed (Figure 1A and B). Expression of Cav-2 was confirmed in DU145 and PC3, whereas Cav-2 was not expressed in LNCap and 22RV1 (Figure 1A and B). Increased expression of AR was observed in LNCap. 22RV1 is an androgen-independent PC cell line; however, weakly expressed AR (Figure 1A and B). These results suggested that Cav-1 and Cav-2 were expressed androgen-independent CRPC cells and may correlate with the downregulation of AR.

\section{Biological role of Cav-I and Cav-2 in PC3 cell line}

In response to the previous result of increased Cav-1-2 expression in PC3 cell line, we performed transient KD of Cav-1 and Cav-2 for the cells and we analyzed the biological activity. Twenty-four hours after transfection, decreased expression of Cav-1 and Cav-2 mRNA and proteins was confirmed by RT-qPCR (Figure 2A). Of interest, expression of Cav-1 was slightly downregulated in Cav-2-KD PC3 cells. Similar phenomenon was observed in our previous study ${ }^{17}$ however, we could not clarify the exact mechanism with this study. We also confirmed that the absence of homologous sequence between Cav-1 and Cav-2-siRNA. The cells were then submitted to MTS assay, cell migration, wound healing assay, and cell invasion assay. No significant difference in cell growth was observed in any cell line (Figure 2B); however, cellular migratory activity was significantly decreased in both Cav-1-KD PC3 and Cav-2-KD PC3 cell lines compared with si-Negative PC3 (Figure 2C). In addition as shown in Figure 2D, significant downregulation of cell motility was observed in Cav-1-KD PC3 and Cav-2-KD PC 3 cell lines by wound healing assay. In contrast, downregulation for invasive capability was faint in both cell lines without statistical significance (Figure 2E).

\section{Downregulation of CAVs for cell motility}

The downregulation of cell motility in Cav-1-KD and Cav-2-KD PC3 suggested that these cells had undergone a mesenchymal-epithelial transition (MET) in vitro. To better understand this change, we assessed the expression 
A

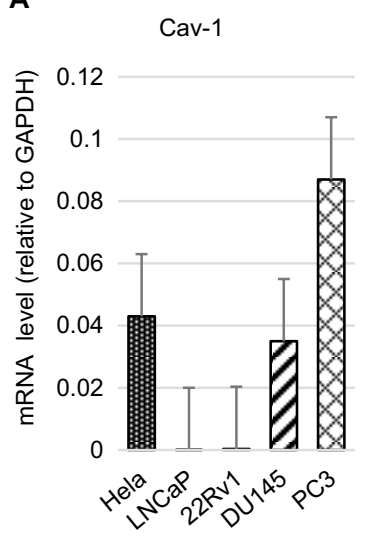

Cav-2

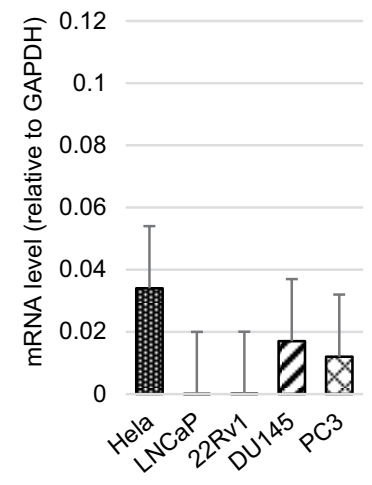

AR

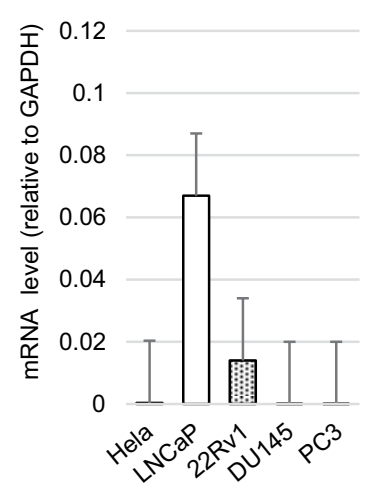

B
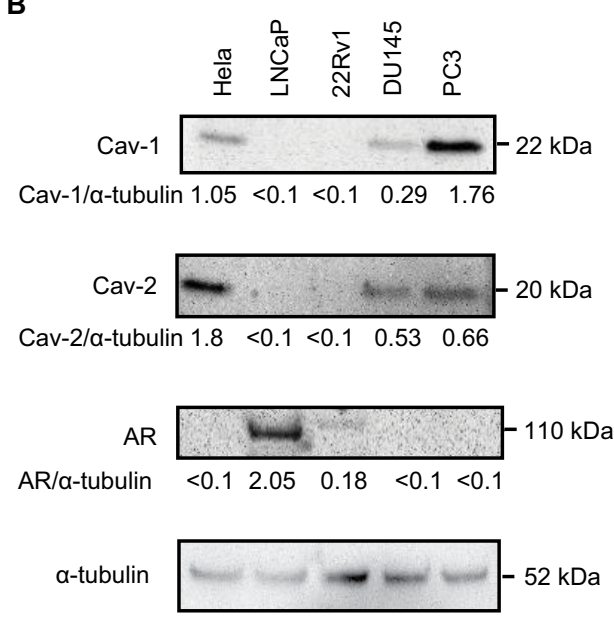

Figure I (A) RT-qPCR analyses of Cav-I, Cav-2, and AR in four PC cell lines (LNCaP, 22RvI, DUI45, and PC3) and cervical cancer cell line (Hela, as a positive control of Cav-I and Cav-2). The relative abundance of Cav-I, Cav-2, and AR was shown as ratios with corresponding GAPDH. (B) Immunoblot analyses of the same cell lines. Ratios to the corresponding $\alpha$-tubulin (Cav-I/ $\alpha$-tubulin, Cav-2/ $\alpha$-tubulin, and AR/ $\alpha$-tubulin) were shown.

Abbreviations: AR, androgen receptor; Cav, caveolin; GAPDH, glyceraldehyde-3-phosphate dehydrogenase; PC, prostate cancer; RT-qPCR, real-time quantitative PCR.

of MET-related molecules in these cells. Following siRNAmediated loss of Cav-1, the level of vimentin mRNA was downregulated (Figure 3). In addition, expression of $\mathrm{N}$-cadherin was slightly decreased. However, no significant difference of E-cadherin, SNAIL, SNAIL2, HAI1, and HAI2 expressions was observed (Figure 3).

Next, we examined the expression of 84 molecules related to cancer metastasis using the $\mathrm{RT}^{2}$ Profiler PCR Array system. As a result, significant downregulation of mannosyl (alpha-1,6)-glycoprotein beta-1,6- $N$-acetyl-glucosaminyltransferase (MGAT) 5, matrix metallopeptidase 13 (MMP13), and V-myc myelocytomatosis viral oncogene homolog 1, lung carcinoma derived (MYCL) was observed in Cav-1-KD PC3 cells (Table 1). In contrast, the expression of MYCL and ETV4 (Ets variant 4) was significantly downregulated in Cav-2-KD PC3 cells compared with control. In addition, fibroblast growth factor receptor 4 (FGFR4) and V-src sarcoma viral oncogene homolog (SRC) were downregulated in Cav-2-KD PC3 cells; however, statistical significance was not achieved. In spite of the downregulation of MYCL, upregulation of MYC was observed in both Cav-1-KD and Cav-2-KD PC3 cells.

\section{Discussion}

In this study, we demonstrated that KD of Cav-1 or Cav-2 caused decreased cell migration and decreased vimentin expression in Cav-1-KD PC3 cells, which may suggest the involvement of cells in the process of MET. Contrary to MET, a shift of epithelial feature toward mesenchymal phenotype may be observed during the progression of cancer. This process is referred to as the epithelial-to-mesenchymal transition (EMT). EMT enhances the motility of cancer cells, and this 
A

Cav-1

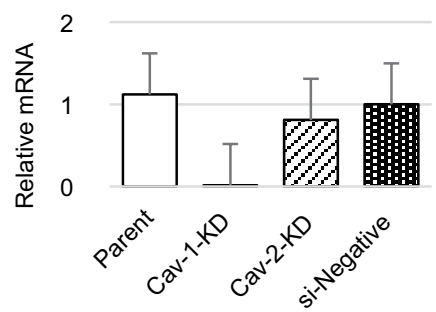

Cav-1

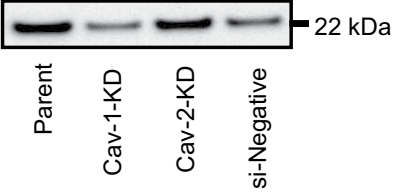

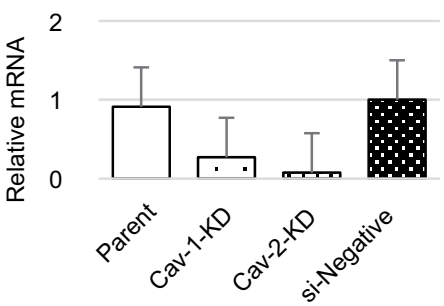

Cav-2

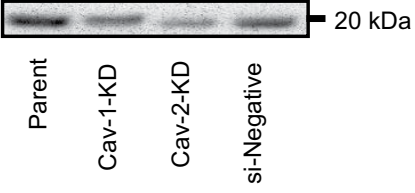

B

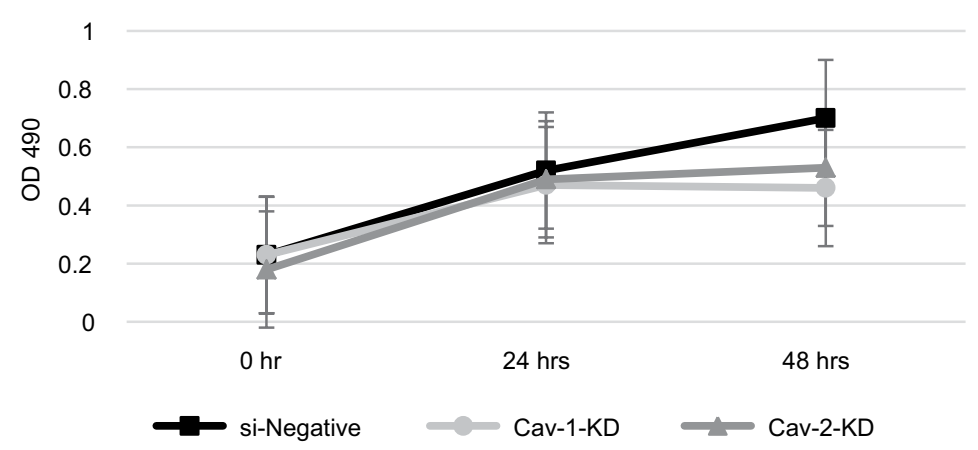

D

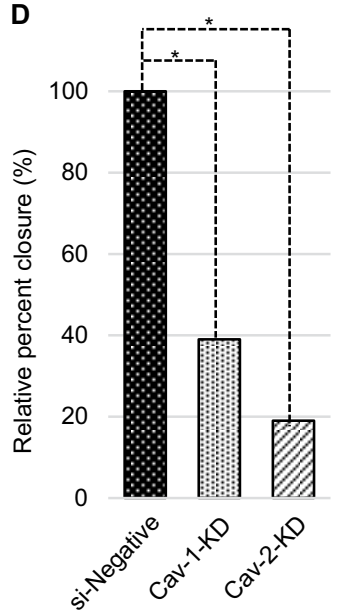

E

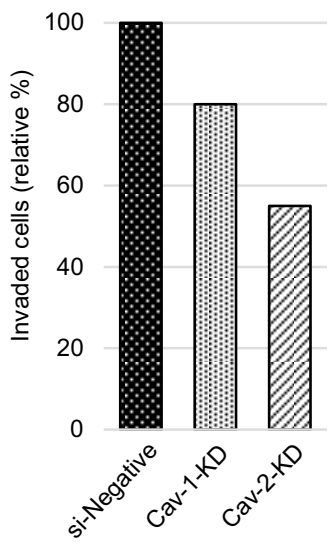

C

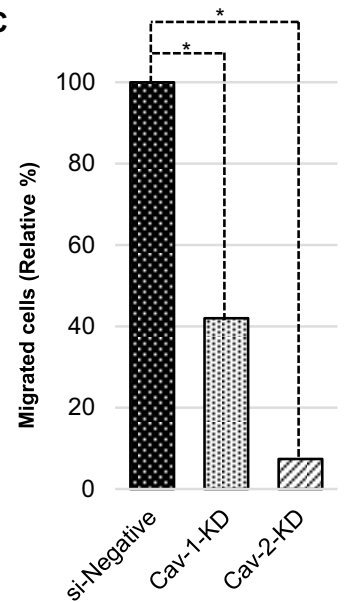

Figure 2 (A) Expression of Cav-I and Cav-2 was confirmed by RT-qPCR and immunoblot analyses in wild-type PC3 (parent), Cav-I-KD PC3, Cav-2-KD PC3, and siNegative-transfected PC3 cell lines. (B) Effect of Cav-1 and Cav-2 expressions on the growth of PC3 cells is analyzed. The cell viability was examined by MTS assay. Each point represents mean \pm SD for six wells. These assays were repeated two times with different batches of transfected cells, and each sample was performed in triplicate. Cell growth of Cav-I-KD and Cav-2-KD PC3 cells was faintly downregulated at 48 hours; however, there is no statistical significance compared with si-Negative PC3 cells. (C) Result of cell migration assay is shown. PC3 cell motility was significantly downregulated by KD of Cav-I and Cav-2. Values are means \pm SD of triplicate experiments. $* P<0.05$, Mann-Whitney $U$ test. (D) The result of wound healing assay reveals downregulation of cell motility of PC3 cells by KD of Cav-I and Cav-2. Values are mean \pm SD of triplicate experiments. ${ }^{*} P<0.05$, Mann-Whitney $U$-test. (E) The result of invasion assay is shown. Invasive activity of $P C 3$ cells was slightly downregulated in both $C a v-I-K D$ and Cav-2-KD PC3 cells; however, statistical significance was not observed.

Abbreviations: Cav, caveolin; KD, knock-down; PC, prostate cancer; RT-qPCR, real-time quantitative PCR; si-Negative, negative control siRNA. 


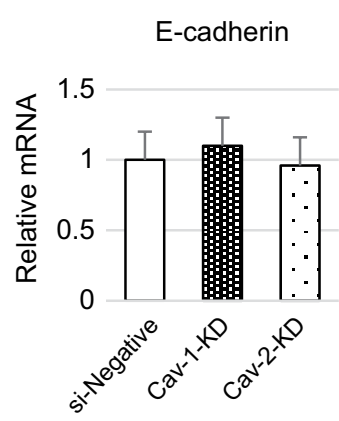

SNAIL

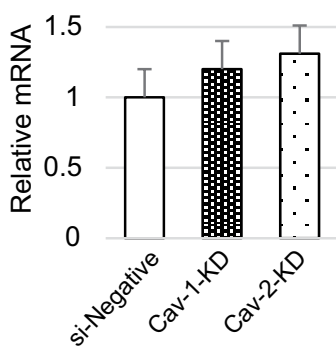

$\mathrm{HAl}-1$

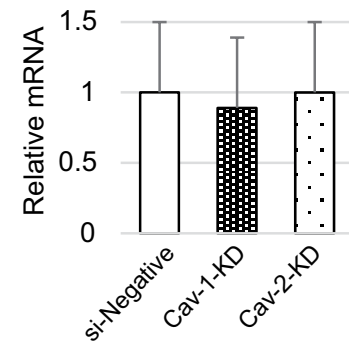

$\mathrm{N}$-cadherin

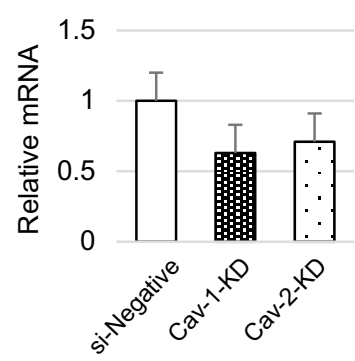

SNAIL2

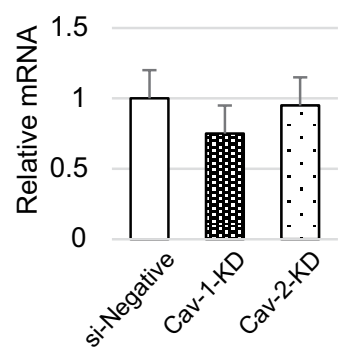

HAI-2

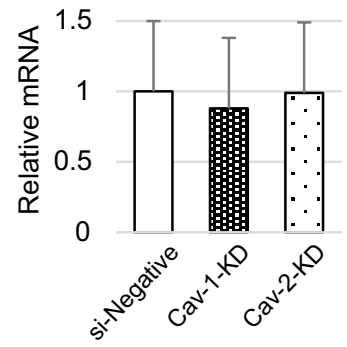

Figure 3 RNA expression of EMT-related molecules was analyzed by RT-qPCR.

Notes: Expression of vimentin was decreased in Cav-I-KD PC3 cells. N-cadherin expression was also slightly decreased in the cells; however, no significant difference of E-cadherin, SNAIL, SNAIL2, EGFR, HAII, and HAI2 expressions was observed.

Abbreviations: Cav, caveolin; EMT, epithelial-to-mesenchymal transition; HAll, hepatocyte growth factor activator inhibitor type I; HAI2, hepatocyte growth factor activator inhibitor type 2; KD, knock-down; PC, prostate cancer; RT-qPCR, real-time quantitative PCR; si-Negative, negative control siRNA.

Table I Expression of molecules related to cancer metastasis in Cav-I-KD and Cav-2-KD PC3 cells, identified by RT ${ }^{2}$ Profiler PCR Array system

\begin{tabular}{|l|l|l|l|}
\hline $\begin{array}{l}\text { Gene } \\
\text { symbol }\end{array}$ & Gene name & $\begin{array}{l}\text { Fold change } \\
\text { Cav-I-KD }\end{array}$ & $\begin{array}{l}\text { Fold change } \\
\text { Cav-2-KD }\end{array}$ \\
\hline MGAT5 & Mannosyl (alpha-I,6-)-glycoprotein beta-I,6-N-acetyl-glucosaminyltransferase & $-2.03^{*}$ & -1.77 \\
MMPI3 & Matrix metallopeptidase I3 (collagenase 3) & $-2.13^{*}$ & 1.12 \\
MYC & V-myc myelocytomatosis viral oncogene homolog (avian) & $2.06^{*}$ & $2.55^{*}$ \\
MYCL & V-myc myelocytomatosis viral oncogene homolog I, lung carcinoma derived (avian) & $-2.3 I^{*}$ & $-2.68^{*}$ \\
ETV4 & Ets variant 4 & -1.65 & $-2.39 *$ \\
FGFR4 & Fibroblast growth factor receptor 4 & -1.64 & -1.95 \\
SRC & V-src sarcoma (Schmidt-Ruppin A-2) viral oncogene homolog (avian) & -1.54 & -1.95 \\
\hline
\end{tabular}

Note: *Twofold or greater change was considered significant.

Abbreviations: Cav, caveolin; KD, knock-down; PC, prostate cancer. 
is considered necessary for cancer invasion. ${ }^{32} \mathrm{~A}$ number of molecular mechanisms of EMT have been reported. ${ }^{32}$ These mechanisms usually involve growth factors, cytokines with their specific receptors, and downstream signaling molecules. Cav-1 is also reported to be implicated in EMT in the progression of prostate, ${ }^{33}$ bladder, ${ }^{34}$ hepatocellular, ${ }^{35}$ and pancreatic cancer. ${ }^{36}$ Liang et $\mathrm{al}^{34}$ demonstrated that elevated Cav-1 expression in bladder cancer was tightly correlated with cancer cell migration and invasion. In this study, the author also revealed that the $\mathrm{KD}$ of Cav-1 reduced the expression of Slug and the ectopic expression of Cav-1-promoted EMT by upregulating Slug expression. In another study, Cav-1 protein expression in pancreatic cancer was analyzed by IHC. ${ }^{36}$ As a result, expression of Cav-1 protein was significantly increased in pancreatic cancer compared with normal tissue. ${ }^{36}$ In addition, cancer cell migration and metastasis were increased in pancreatic cancer cells with elevated expression of Cav-1. The cancer cells show typical morphological changes in EMT with decreased E-cadherin expression. In colorectal cancer, overexpression of Cav-1 was correlated with a decreased expression of E-cadherin, which may indicate that Cav-1 was a negative regulator of E-cadherin in the progression of colorectal cancer. ${ }^{37}$ Vimentin is recognized as a canonical mesenchymal marker, which is a member of the type III intermediate filament protein family. ${ }^{38,39}$ Vimentin is an important component of cellular cytoskeleton and is reported to play a significant role in maintaining cell-shape, integrity, cell adhesion, and migration. ${ }^{38,39}$ Increased expression of vimentin has been reported in the invasive phenotype of several malignant tumors, including prostate, gastrointestinal, breast, lung, cervical cancer, and renal cell carcinoma, ${ }^{38,39}$ and to be associated with increased capacity for cell motility and invasiveness in the process of EMT. ${ }^{38,39}$ Our results suggested that KD of Cav-1 may contribute to the suppression of cell migration through the downregulation of vimentin, which suggests that the cells may be involved in the initial process of MET; however, we could not confirm increased expression of E-cadherin in this study.

In contrast, the result of PCR array revealed the downregulation of MGAT5, MMP13, and MYCL in cav-1-KD PC3 cells. MGAT5, which belongs to the glycosyltransferase family, is reported to be a major enzyme involved in the regulation of glycoprotein oligosaccharide biosynthesis. ${ }^{40,41}$ It has been reported that overexpression of MGAT5 resulted in the loss of contact inhibition, increased cell motility and morphological transformation in epithelial cells, and enhanced metastasis in breast cancer cells. ${ }^{40,41}$ MMP13 is well known as a member of the collagenase subfamily of MMPs with the potential to degrade extracellular matrix components. ${ }^{42}$ In cancer, MMP13 is reported to be positively associated with local invasion, cell migration, vascular infiltration, and metastasis. ${ }^{42} \mathrm{MYC}$ is a major cell regulator in many processes including cell cycle entry, ribosome biogenesis, and metabolism. ${ }^{43,44}$ Deregulation of MYC associated with tumor aggressiveness, metastatic potential, therapeutic resistance, and poor patient outcome has been reported in various cancers. ${ }^{43,44} \mathrm{MYCL}$ was reported to correlate with the progression of small cell lung cancer. ${ }^{45}$ With the exception of increased MYC expression, these data support our migration assay results; however, further examination will be required to clarify the exact functional mechanism of Cav-1 in the progression of PC.

Cav-2 was discovered as a $20 \mathrm{kDa}$ protein co-purified with adipocyte-derived caveolar membranes. ${ }^{7} \mathrm{Cav}-2$-encoded gene is located on human chromosome $7 \mathrm{q} 31.1 .^{7} \mathrm{Cav}-2$ can form a stable hetero-oligomeric complex of 14-16 molecules into a large macromolecular complex, which defines caveolar architecture at the plasma membrane. ${ }^{6,7}$ In the physiological condition, Cav-2 is reported to express and play with Cav-1.$^{6,7}$ In cancer, increased Cav-2 expression has been documented in esophageal carcinoma and urothelial carcinoma, as well as in the cell lines of glioma, cervical cancer, lung adenocarcinoma, and breast cancer. ${ }^{7}$ Its function in cancer progression varies by cancer cell line. ${ }^{7}$ We were unable to identify the exact focus of this phenomenon by RT-qPCR of EMT-related molecules in Cav-2-KD PC3 cells. However, several candidate molecules were detected in PCR array. ETV4 is a transcription factor belonging to the ETS-oncogene family. ${ }^{46}$ Overexpression of ETV4 was reported to be associated with advanced and more aggressive forms of tumor with worse prognosis. ${ }^{46}$ In addition, ETV4 positively correlates with cancer cell motility, invasiveness, EMT, and tumorigenesis. ${ }^{46}$ Activated SRC and FGFR4 are also reported to influence cancer cell proliferation, migration, and invasion through the specific signaling axis. ${ }^{47,48}$ From the above, downregulated cell motility in Cav-2-KD PC3 cells may be caused by decreased expression of ETV4, SRC, or FGFR 4. ${ }^{46-48}$ Therefore, we are considering additional analysis to clarify the mechanism.

In $\mathrm{PC}$, it has been reported that ADT directory induces $\mathrm{EMT}^{49}$ and that decreased E-cadherin expression is observed in CRPC. ${ }^{50}$ These data suggested that EMT would be upregulated in CRPC. In contrast, increased expression of Cav-1 in CRPC has been reported and several reports indicate Cav-1-induced progression of EMT. ${ }^{33}$ Therefore, inverse 
expression of $\mathrm{AR}$ and $\mathrm{Cav}-1$ may have an important role in CRPC progression through EMT.

\section{Conclusion}

KD of Cav-1 and Cav-2 downregulates the cell motility of PC3 cells. Expression of vimentin was decreased in Cav1-KD PC3 cells. In addition, analysis of PCR array revealed decreased expression of MGAT5, MMP13, and MYCL in Cav-1-KD PC3 and ETV4, FGFR4, and SRC in Cav-2-KD PC3. Cav-induced regulation of these molecules may have an important role in PC3 cell motility. Cav-1 and Cav-2 may positively contribute to the upregulation of CRPC cell migration.

\section{Disclosure}

The authors report no conflicts of interest in this work.

\section{References}

1. Siegel RL, Miller KD, Jemal A. Cancer statistics, 2015. CA Cancer J Clin. 2015;65(1):5-29.

2. Cancer Registration Committee of the Japanese Urological Association Clinicopathological statistics on registered prostate cancer patients in Japan. report from the Japanese Urological Association. Int J Urol. 2000;2005(12):46-61.

3. Hinotsu S, Akaza H, Usami M et al. Current status of endocrine therapy for prostate cancer in Japan - analysis of primary androgen deprivation therapy on the basis of data collected by J-CaP. Jpn J Clin Oncol. 2007;37:775-781

4. Cooperberg MR, Hinotsu S, Namiki M, et al. Risk assessment among prostate cancer patients receiving primary androgen deprivation therapy. J Clin Oncol. 2009;27(26):4306-4313.

5. Hotte SJ, Saad F. Current management of castrate-resistant prostate cancer. Curr Oncol. 2010;17(Suppl 2):72-79.

6. Fridolfsson HN, Roth DM, Insel PA, Patel HH. Regulation of intracellular signaling and function by caveolin. Faseb J. 2014;28(9):3823-3831.

7. Gupta R, Toufaily C, Annabi B. Caveolin and cavin family members: dual roles in cancer. Biochimie. 2014;107 Pt B:188-202.

8. Sonnino S, Prinetti A. Sphingolipids and membrane environments for caveolin. FEBS Lett. 2009;583(4):597-606.

9. Chen D, Che G. Value of caveolin-1 in cancer progression and prognosis: Emphasis on cancer-associated fibroblasts, human cancer cells and mechanism of caveolin-1 expression (Review). Oncol Lett. 2014;8(4):1409-1421.

10. Burgermeister E, Liscovitch M, Röcken C, Schmid RM, Ebert MP. Caveats of caveolin-1 in cancer progression. Cancer Lett. 2008;268(2): 187-201.

11. Ando T, Ishiguro H, Kimura M, et al. The overexpression of caveolin-1 and caveolin-2 correlates with a poor prognosis and tumor progression in esophageal squamous cell carcinoma. Oncol Rep. 2007;18(3): 601-609.

12. Sagara Y, Mimori K, Yoshinaga K, et al. Clinical significance of Caveolin-1, Caveolin-2 and HER2/neu mRNA expression in human breast cancer. Br J Cancer. 2004;91(5):959-965.

13. Nimri L, Barak H, Graeve L, Schwartz B. Restoration of caveolin-1 expression suppresses growth, membrane-type-4 metalloproteinase expression and metastasis-associated activities in colon cancer cells. Mol Carcinog. 2013;52(11):859-870.

14. Davidson B, Nesland JM, Goldberg I, et al. Caveolin-1 expression in advanced-stage ovarian carcinoma--a clinicopathologic study. Gynecol Oncol. 2001;81(2):166-171.
15. Wiechen K, Sers C, Agoulnik A, et al. Down-regulation of caveolin-1, a candidate tumor suppressor gene, in sarcomas. Am J Pathol. 2001;158(3):833-839.

16. Karuppiah K, Druhan LJ, Chen CA, et al. Suppression of eNOS-derived superoxide by caveolin-1: a biopterin-dependent mechanism. Am J Physiol Heart Circ Physiol. 2011;301(3):H903-H911.

17. Sugie S, Mukai S, Yamasaki K, Kamibeppu T, Tsukino H, Kamoto T. Significant association of caveolin- 1 and caveolin-2 with prostate cancer progression. Cancer Genomics Proteomics. 2015;12(6):391-396.

18. Yang G, Goltsov AA, Ren C, et al. Caveolin-1 upregulation contributes to c-Myc-induced high-grade prostatic intraepithelial neoplasia and prostate cancer. Mol Cancer Res. 2012;10(2):218-229.

19. Williams TM, Hassan GS, Li J, et al. Caveolin-1 promotes tumor progression in an autochthonous mouse model of prostate cancer: genetic ablation of Cav-1 delays advanced prostate tumor development in tramp mice. J Biol Chem. 2005;280(26):25134-25145.

20. Sugie S, Mukai S, Tsukino H, et al. Increased plasma caveolin-1 levels are associated with progression of prostate cancer among Japanese men. Anticancer Res. 2013;33(5):1893-1897.

21. Suzuoki M, Miyamoto M, Kato K, et al. Impact of caveolin-1 expression on prognosis of pancreatic ductal adenocarcinoma. Br J Cancer. 2002;87(10):1140-1144.

22. Chatterjee M, Ben-Josef E, Thomas DG, et al. caveolin-1 is associated with tumor progression and confers a multi-modality resistance phenotype in pancreatic cancer. Sci Rep. 2015;5(12):10867.

23. Bender FC, Reymond MA, Bron C, Quest AF. Caveolin-1 levels are down-regulated in human colon tumors, and ectopic expression of caveolin-1 in colon carcinoma cell lines reduces cell tumorigenicity. Cancer Res. 2000;60(20):5870-5878.

24. van den Eynden GG, van Laere SJ, van der Auwera I, et al. Overexpression of caveolin-1 and - 2 in cell lines and in human samples of inflammatory breast cancer. Breast Cancer Res Treat. 2006;95(3):219-228.

25. Kato K, Hida Y, Miyamoto M, et al. Overexpression of caveolin-1 in esophageal squamous cell carcinoma correlates with lymph node metastasis and pathologic stage. Cancer. 2002;94(4):929-933.

26. Ando T, Ishiguro H, Kimura M, et al. The overexpression of caveolin-1 and caveolin-2 correlates with a poor prognosis and tumor progression in esophageal squamous cell carcinoma. Oncol Rep. 2007;18(3):601-609.

27. Hu YC, Lam KY, Law S, Wong J, Srivastava G. Profiling of differentially expressed cancer-related genes in esophageal squamous cell carcinoma (ESCC) using human cancer cDNA arrays: overexpression of oncogene MET correlates with tumor differentiation in ESCC. Clin Cancer Res. 2001;7(11):3519-3525.

28. Fong A, Garcia E, Gwynn L, Lisanti MP, Fazzari MJ, Li M. Expression of caveolin-1 and caveolin-2 in urothelial carcinoma of the urinary bladder correlates with tumor grade and squamous differentiation. $\mathrm{Am}$ J Clin Pathol. 2003;120(1):93-100.

29. Yuan G, Regel I, Lian F, et al. WNT6 is a novel target gene of caveolin-1 promoting chemoresistance to epirubicin in human gastric cancer cells. Oncogene. 2013;32(3):375-387.

30. Li M, Chen H, Diao L, Zhang Y, Xia C, Yang F. Caveolin-1 and VEGF-C promote lymph node metastasis in the absence of intratumoral lymphangiogenesis in non-small cell lung cancer. Tumori. 2010;96(5):734-743.

31. Nasu Y, Timme TL, Yang G, et al. Suppression of caveolin expression induces androgen sensitivity in metastatic androgen-insensitive mouse prostate cancer cells. Nat Med. 1998;4(9):1062-1064.

32. Cheng H, Fukushima T, Takahashi N, Tanaka H, Kataoka H. Hepatocyte growth factor activator inhibitor type 1 regulates epithelial to mesenchymal transition through membrane-bound serine proteinases. Cancer Res. 2009;69(5):1828-1835.

33. Zhang X, Ling MT, Wang Q, et al. Identification of a novel inhibitor of differentiation-1 (ID-1) binding partner, caveolin-1, and its role in epithelial-mesenchymal transition and resistance to apoptosis in prostate cancer cells. J Biol Chem. 2007;282(46):33284-33294.

34. Liang W, Hao Z, Han JL, Zhu DJ, Jin ZF, Xie WL. CAV-1 contributes to bladder cancer progression by inducing epithelial-to-mesenchymal transition. Urol Oncol. 2014;32(6):855-863. 
35. Meyer C, Dzieran J, Liu Y, et al. Distinct dedifferentiation processes affect caveolin-1 expression in hepatocytes. Cell Commun Signal. 2013;11(1):6.

36. Huang C, Qiu Z, Wang L, et al. A novel FoxM1-caveolin signaling pathway promotes pancreatic cancer invasion and metastasis. Cancer Res. 2012;72(3):655-665.

37. Zhang J, Zhang K, Zhou L, et al. Expression and potential correlation among Forkhead box protein M1, Caveolin-1 and E-cadherin in colorectal cancer. Oncol Lett. 2016;12(4):2381-2388.

38. Satelli A, Li S. Vimentin as a potential molecular target in cancer therapy Or Vimentin, an overview and its potential as a molecular target for cancer therapy. Cell Mol Life Sci. 2011;68(18):3033-3046.

39. Xu Z, Bian $\mathrm{H}$, Zhang F, et al. URI promotes the migration and invasion of human cervical cancer cells potentially via upregulation of vimentin expression. Am J Transl Res. 2017;9(6):3037.

40. Wang $\mathrm{X}, \mathrm{He} \mathrm{H}$, Zhang $\mathrm{H}$, et al. Clinical and prognostic implications of $\beta 1,6-\mathrm{N}$-acetylglucosaminyltransferase $\mathrm{V}$ in patients with gastric cancer. Cancer Sci. 2013;104(2):185-193.

41. Granovsky M, Fata J, Pawling J, Muller WJ, Khokha R, Dennis JW. Suppression of tumor growth and metastasis in Mgat5-deficient mice. Nat Med. 2000;6(3):306-312.

42. Osako Y, Seki N, Kita Y, et al. Regulation of MMP13 by antitumor microRNA-375 markedly inhibits cancer cell migration and invasion in esophageal squamous cell carcinoma. Int JOncol. 2016;49(6):2255-2264.
43. Schick M, Habringer S, Nilsson JA, Keller U. Pathogenesis and therapeutic targeting of aberrant MYC expression in haematological cancers. Br J Haematol. 2017;179(5):724-738.

44. Kalkat M, de Melo J, Hickman KA, et al. MYC Deregulation in Primary Human Cancers. Genes. 2017;8(6):151.

45. Kato F, Fiorentino FP, Alibés A. MYCL is a target of a BET bromodomain inhibitor, JQ1, on growth suppression efficacy in small cell lung cancer cells. Oncotarget. 2017;7(47):77378.

46. Tyagi N, Deshmukh SK, Srivastava SK, et al. ETV4 Facilitates CellCycle Progression in Pancreatic Cells through Transcriptional Regulation of Cyclin D1. Mol Cancer Res. 2018;16(2):187-196.

47. Wang S, Ding Z. Fibroblast growth factor receptors in breast cancer. Tumor Biol. 2017;39(5):1010428317698370.

48. Anbalagan M, Moroz K, Ali A, Carrier L, Glodowski S, Rowan BG. Subcellular localization of total and activated Src kinase in African American and Caucasian breast cancer. PLoS One. 2012;7(3):e33017.

49. Sun Y, Wang BE, Leong KG, et al. Androgen deprivation causes epithelial-mesenchymal transition in the prostate: implications for androgen-deprivation therapy. Cancer Res. 2012;72(2):527-536.

50. Figiel S, Vasseur C, Bruyere F, Rozet F, Maheo K, Fromont G. Clinical significance of epithelial-mesenchymal transition markers in prostate cancer. Hum Pathol. 2017;61:26-32.
Research and Reports in Urology

\section{Publish your work in this journal}

Research and Reports in Urology is an international, peer-reviewed, open access journal publishing original research, reports, editorials, reviews and commentaries on all aspects of adult and pediatric urology in the clinic and laboratory including the following topics: Pathology, pathophysiology of urological disease; Investigation and treatment of

\section{Dovepress}

urological disease; Pharmacology of drugs used for the treatment of urological disease. The manuscript management system is completely online and includes a very quick and fair peer-review system, which is all easy to use. Visit http://www.dovepress.com/testimonials.php to read real quotes from published authors. 